\title{
A review on mycotoxins in food and feed : Malaysia case study
}

\begin{abstract}
Fungi are distributed worldwide and can be found in various foods and feedstuffs from almost every part of the world. Mycotoxins are secondary metabolites produced by some fungal species and may impose food safety risks to human health. Among all mycotoxins, aflatoxins (AFs), ochratoxin A (OTA), trichothecenes, deoxynivalenol (DON and T-2 toxin), zearalenone (ZEN), and fumonisins (FMN) have received much attention due to high frequency and severe health effects in humans and animals. Malaysia has heavy rainfall throughout the year, high temperatures $\left(28\right.$ to $\left.31{ }^{\circ} \mathrm{C}\right)$, and high relative humidity $(70 \%$ to $80 \%$ during wet seasons). Stored crops under such conditions can easily be contaminated by mycotoxin-producing fungi. The most important mycotoxins in Malaysian foods are AFs, OTA, DON, ZEN, and FMN that can be found in peanuts, cereal grains, cocoa beans, and spices. AFs have been reported to occur in several cereal grains, feeds, nuts, and nut products consumed in Malaysia. Spices, oilseeds, milk, eggs, and herbal medicines have been reported to be contaminated with AFs (lower than the Malaysian acceptable level of $35 \mathrm{ng} / \mathrm{g}$ for total AFs). OTA, a possible human carcinogen, was reported in cereal grains, nuts, and spices in Malaysian market. ZEN was detected in Malaysian rice, oat, barley, maize meal, and wheat at different levels. DON contamination, although at low levels, was reported in rice, maize, barley, oat, wheat, and wheat-based products in Malaysia. FMN was reported in feed and some cereal grains consumed in Malaysia. Since some food commodities are more susceptible than others to fungal growth and mycotoxin contamination, more stringent prevention and control methods are required.
\end{abstract}

Keyword: Aflatoxins; Ochratoxin A; Trichothecenes; Deoxynivalenol; Zearalenone; Fumonisins; Cereals; Nuts; Spices 